\title{
WATER ICE IN THE KUIPER BELT
}

\author{
M. E. Brown ${ }^{1}$, E. L. Schaller ${ }^{2,3}$, and W. C. Fraser $^{1}$ \\ ${ }^{1}$ Division of Geological and Planetary Sciences, California Institute of Technology, Pasadena, CA 91125, USA; mbrown@ caltech.edu \\ 2 NASA Dryden Aircraft Operations Facility, Palmdale, CA 93550, USA \\ ${ }^{3}$ National Suborbital Education and Research Center, University of North Dakota, Grand Forks, ND 85202, USA \\ Received 2012 February 7; accepted 2012 April 10; published 2012 May 14
}

\begin{abstract}
We examine a large collection of low-resolution near-infrared spectra of Kuiper Belt objects (KBOs) and centaurs in an attempt to understand the presence of water ice in the Kuiper Belt. We find that water ice on the surface of these objects occurs in three separate manners: (1) Haumea family members uniquely show surfaces of nearly pure water ice, presumably a consequence of the fragmentation of the icy mantle of a larger differentiated proto-Haumea; (2) large objects with absolute magnitudes of $H<3$ (and a limited number to $H=4.5$ ) have surface coverings of water ice-perhaps mixed with ammonia - that appears to be related to possibly ancient cryovolcanism on these large objects; and (3) smaller KBOs and centaurs which are neither Haumea family members nor cold-classical KBOs appear to divide into two families (which we refer to as "neutral" and "red"), each of which is a mixture of a common nearly neutral component and either a slightly red or very red component that also includes water ice. A model suggesting that the difference between neutral and red objects due to formation in an early compact solar system either inside or outside, respectively, of the $\sim 20$ AU methanol evaporation line is supported by the observation that methanol is only detected on the reddest objects, which are those which would be expected to have the most of the methanol containing mixture.
\end{abstract}

Key words: astrochemistry - Kuiper belt: general - planets and satellites: composition - planets and satellites: formation

Online-only material: color figures

\section{INTRODUCTION}

The Kuiper Belt is composed of low-temperature remnants of the outer regions of the protoplanetary disk which never became incorporated into planets. By analogy to short-period comets, which are derived from the Kuiper Belt, and from cosmochemical considerations, it is expected that water ice is a major constituent of the composition of Kuiper Belt objects (KBOs). That water ice was the first clearly identified constituent on the surfaces of small KBOs and of centaurs (former KBOs which are currently on short-lived planet-crossing orbits) was thus not a surprise (Brown et al. 1998, 1999).

For a large majority of KBOs that have been studied, water ice remains the only identifiable surface constituent, even though the detectable absorption features are so small that in most cases it is clear that water ice is a relatively minor component of the surface (Barkume et al. 2006; Guilbert et al. 2009). One major exception is Haumea and its satellites and collisional family, which appear to have surfaces composed of nearly pure water ice, thought to be exposed when the differentiated icy mantle of the proto-Haumea was removed in a giant impact (Brown et al. 2007). The other major exception is the largest KBOs, which are cold and massive enough to maintain volatile atmospheres and frosts over the age of the solar system (Schaller \& Brown 2007), and whose bedrock is covered and thus unobservable. For the majority of KBOs with water ice present in the spectrum, little connection has been made between the water ice visible on the surface and any other properties of the KBOs. No simple correlation appears between dynamical or color properties and water ice absorption (Barkume et al. 2006; Guilbert et al. 2009), so emphasis has instead mostly been on detailed modeling to determine surface constituents (i.e., Barucci et al. 2011) and quantify the absence or presence of ice.
Here we examine the moderate-sized and smaller KBOs and examine water and other ices in detail. The goal is to examine these objects as a class, rather than perform detailed modeling of individual objects, in the hope of statistically understanding the causes and states of ices on these objects.

\section{OBSERVATIONS AND ANALYSIS}

In order to examine the properties of ices in the Kuiper Belt, we assemble a nearly uniform set of low-resolution $(\lambda / \Delta \lambda \sim$ 160) $1.5-2.4 \mu \mathrm{m}$ reflectance spectra, almost all obtained using NIRC - the first-generation near-infrared spectrograph at the Keck 1 telescope (Matthews \& Soifer 1994). Spectra were collected from the surveys of Brown (2000) and Barkume et al. (2008). We also include the NIRC spectrum of Charon (Brown \& Calvin 2000) obtained with the identical instrument, and the only available spectrum of 2007 OR10, obtained with the FIRE spectrograph at the Magellan telescope (Brown et al. 2011a).

To augment this existing sample, we obtained new spectra of $15 \mathrm{KBOs}$ and centaurs with the NIRC spectrograph on the Keck telescope until the spectrograph was retired in 2009. The observations and data reduction were performed identically to the original Keck surveys, and details are given in Table 1. The absolute magnitudes shown in the table are taken from the Minor Planet Center, which is the only compilation which includes all of the objects in our sample. Such absolute magnitudes appear to be systematically biased compared to well-measured samples (Romanishin \& Tegler 2005), so, for relative consistency, we use only the MPC magnitudes even though more accurate measurements are available for some of the objects. Random uncertainties in absolute magnitude are not reported by the Minor Planet Center but, by comparison to Romanishin \& Tegler (2005) appear to be less than $0.3 \mathrm{mag}$. For objects with observations at multiple epochs we took the average of 
Table 1

Log of Observations

\begin{tabular}{|c|c|c|c|c|}
\hline Object & $\begin{array}{l}\text { Date } \\
\text { (UT) }\end{array}$ & $\begin{array}{l}\text { Exp. Time } \\
\text { (s) }\end{array}$ & Airmass & Calibrator \\
\hline 19521 Chaos (1998 WH24) & 2007 Sep 23 & 4000 & $1.02-1.00$ & HD283798 \\
\hline 145451 (2005 RM43) & $2007 \mathrm{Sep} 24$ & 7000 & $1.15-1.06$ & U Peg \\
\hline \multirow[t]{5}{*}{248835 (2006 SX368) } & 2007 Sep 24 & 3400 & $1.02-1.04$ & V Aql \\
\hline & 2008 May 20 & 1200 & $1.54-1.06$ & HD183186 \\
\hline & 2008 May 21 & 800 & $1.12-1.02$ & HD183186 \\
\hline & 2008 Oct 20 & 1000 & $1.00-1.01$ & HD211839 \\
\hline & 2008 Oct 21 & 1000 & $1.02-1.01$ & G 185 \\
\hline 73480 (2002 PN34) & 2007 Sep 24 & 6000 & $1.05-1.07$ & $(\mathrm{~V} * 00 \mathrm{Aql})$ \\
\hline \multirow[t]{2}{*}{44594 (1999 OX3) } & 2007 Sep 25 & 2600 & $1.18-1.35$ & HD 203812 \\
\hline & 2008 May 22 & 400 & $1.51-1.38$ & HD209251 \\
\hline 145486 (2005 UJ438) & 2007 Sep 25 & 1200 & $1.09-1.05$ & LHS1365 \\
\hline $120061(2003$ CO1) & 2008 May 22 & 800 & $1.08-1.50$ & HD154086, HD133011, HD 132724 \\
\hline \multirow[t]{2}{*}{174567 (2003 MW12) } & 2008 May 22 & 800 & $1.14-1.30$ & HD133011 \\
\hline & 2009 May 6 & 6000 & $1.10-1.34$ & HD170363, BD-02 4024 \\
\hline \multirow[t]{2}{*}{32532 Thereus (2001 PT13) } & 2008 Oct 17 & 3400 & $1.11-1.04$ & G $18-5$ \\
\hline & 2008 Oct 18 & 4000 & $1.01-1.07$ & HD19061 \\
\hline \multirow[t]{2}{*}{ (2007 UM126) } & 2008 Oct 17 & 6400 & $1.18-1.04$ & LHS 1231, HD 27834 \\
\hline & 2008 Oct 19 & 4000 & $1.17-1.08$ & HD 27834 \\
\hline \multirow{2}{*}{229762 (2007 UK126) } & 2008 Oct 19 & 2000 & $1.14-1.22$ & HD19061, HD233399 \\
\hline & 2009 Sep 30 & 8000 & $1.09-1.14$ & HD289960, HD287530 \\
\hline \multirow{2}{*}{90482 Orcus (2004DW) } & 2009 May 6 & 3000 & $1.14-1.16$ & HD95259 \\
\hline & 2009 May 8 & 5000 & $1.11-1.22$ & HD101731 \\
\hline 90568 (2004 GV9) & 2009 May 6 & 5000 & $1.61-1.49$ & HD136122 \\
\hline \multirow[t]{2}{*}{42355 Typhon (2002 CR46) } & 2009 May 7 & 5000 & $1.05-1.25$ & $\mathrm{BD}+003226$ \\
\hline & 2009 May 9 & 6000 & $1.05-1.07$ & $\mathrm{BD}+003226$ \\
\hline \multirow[t]{2}{*}{2008 AP129 } & 2008 Oct 19 & 1000 & $1.34-1.28$ & $\mathrm{BD}+45-1586$ \\
\hline & 2009 Oct 1 & 9000 & $1.12-1.15$ & HD289960, HD287530 \\
\hline
\end{tabular}

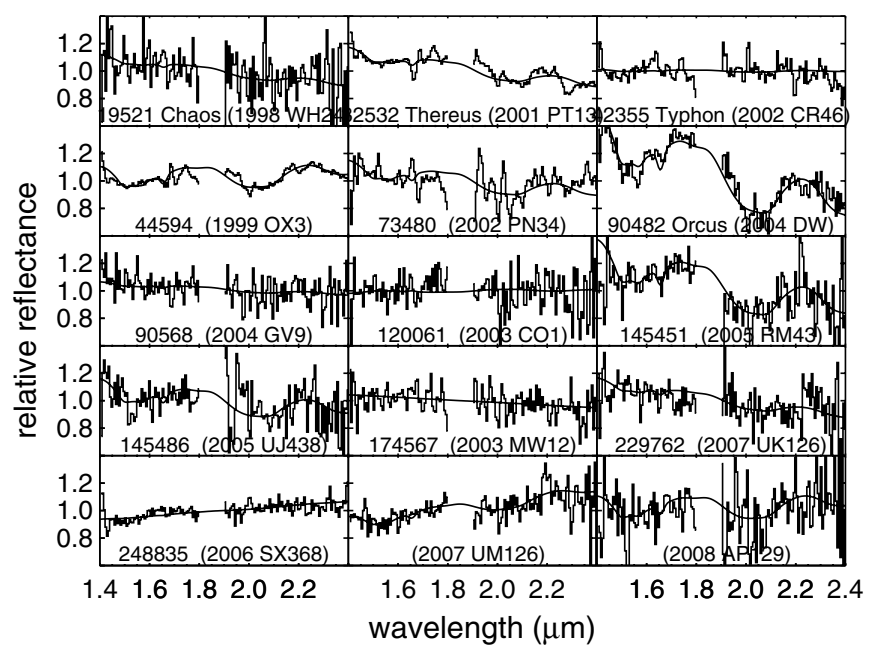

Figure 1. Newly acquired spectra of KBOs and centaurs. The best-fit water ice plus sloped continuum model is shown for each spectrum. All spectra are normalized to a median value of one across the observed wavelength range.

the individual spectra. The KBO 19521 Chaos (1998 WH24) appeared unusual in the previous survey, so it was reobserved to determine if it has a unique spectral type or if the observations were faulty. The new spectrum closely resembles other KBO spectra, so we assume the previous spectrum was in error and only retain the new spectrum. Figure 1 shows all of the newly obtained spectra.

The full spectral sample (see Table 2) includes 64 objects. To examine ices on the smaller objects, we remove from consideration objects known to be part of Haumea's collisional family (Brown et al. 2007; Haumea, 1995 SM55, 2002 TX300,
2003 OP32, 2005 RR43) and also the largest objects for which methane dominates the spectrum (Eris, Pluto, Makemake, and Sedna). A total of 57 objects remain in the sample at this point.

We are interested in characterizing the amount of water ice absorption present in each of the spectra, but spectral models give non-unique results for the fraction of water ice present on a surface. Differences in assumed grain size, type of mixing, and non-water ice components can lead to orderof-magnitude or greater variations in the derived water ice abundance. Frequently, a measurement of the depth of the $2 \mu \mathrm{m}$ water absorption feature is used as a model-independent proxy for the amount of water ice present (Brown 2000; Jewitt \& Luu 2001; Barucci et al. 2011), but this method ignores much of the information contained in a broader spectrum. Barkume et al. (2008), therefore, developed a simple parametric method to fit a spectrum. While the derived parameters cannot be directly converted to a composition, they uniquely describe the basic characteristics of the spectrum of the object and thus facilitate quantitative comparison. We use a modified version of that parameterization here.

We assume that in the region from 1.4 to $2.4 \mu \mathrm{m}$ the spectra of these KBOs can be modeled to the accuracy of the currently available data by a simple model consisting of a linear mixture of a water ice spectrum and a sloped continuum. In our parameterization, the model spectrum, $s[\lambda]$, is simply given by

$s[\lambda]=f_{\text {water }} s_{\text {water }}[\lambda]+\left(1-f_{\text {water }}\right)\left[m_{\text {cont }}(\lambda-1.74 \mu \mathrm{m})+.49\right]$,

where $f_{\text {water }}$ is a parameter which scales the amount of water ice versus continuum, $s_{\text {water }}$ is a modeled water ice spectrum, $m_{\text {cont }}$ is the slope of the continuum added to the water ice spectrum, and $\lambda$ is the wavelength in $\mu \mathrm{m}$. For a modeled water ice spectrum we used optical constants from Grundy \& Schmitt (1998) at 
Table 2

Characteristics of Observed KBOs and Centaurs

\begin{tabular}{|c|c|c|c|c|c|c|c|c|}
\hline Object & $\begin{array}{c}a \\
(\mathrm{AU})\end{array}$ & $e$ & $\begin{array}{c}i \\
(\operatorname{deg})\end{array}$ & $\begin{array}{c}H \\
(\mathrm{mag})\end{array}$ & $f_{\text {water }}$ & Uncertainty & $m_{\text {cont }}$ & Uncertainty \\
\hline 2060 Chiron (1977 UB) & 13.7 & 0.38 & 6.9 & 6.3 & -0.00 & 0.01 & 0.08 & 0.00 \\
\hline 5145 Pholus (1992 AD) & 20.3 & 0.57 & 24.7 & 7.1 & 0.13 & 0.01 & -0.12 & 0.00 \\
\hline 8405 Asbolus (1995 GO) & 18.1 & 0.62 & 17.6 & 9.0 & -0.00 & 0.04 & -0.00 & 0.00 \\
\hline 10199 Chariklo (1997 CU26) & 15.7 & 0.17 & 23.4 & 6.6 & 0.19 & 0.01 & 0.04 & 0.00 \\
\hline 15875 (1996 ТР66) & 39.7 & 0.34 & 5.7 & 6.9 & 0.00 & 0.08 & -0.21 & 0.05 \\
\hline 20000 Varuna (2000 WR106) & 43.0 & 0.06 & 17.2 & 3.6 & 0.01 & 0.02 & -0.09 & 0.00 \\
\hline 24835 (1995 SM55) & 42.0 & 0.11 & 27.0 & 4.8 & 0.83 & 0.08 & 0.17 & 0.24 \\
\hline 26181 (1996 GQ21) & 92.4 & 0.59 & 13.4 & 5.2 & 0.07 & 0.04 & -0.10 & 0.03 \\
\hline 26375 (1999 DE9) & 55.2 & 0.42 & 7.6 & 5.1 & 0.10 & 0.05 & -0.18 & 0.03 \\
\hline 28978 Ixion (2001 KX76) & 39.5 & 0.25 & 19.7 & 3.3 & 0.04 & 0.05 & -0.05 & 0.03 \\
\hline 29981 (1999 TD10) & 99.4 & 0.88 & 6.0 & 8.7 & 0.06 & 0.02 & -0.07 & 0.00 \\
\hline 31824 Elatus (1999 UG5) & 11.8 & 0.38 & 5.2 & 10.1 & 0.03 & 0.02 & 0.06 & 0.00 \\
\hline 33340 (1998 VG44) & 39.5 & 0.26 & 3.0 & 6.5 & 0.06 & 0.18 & -0.14 & 0.10 \\
\hline 38628 Huya (2000 EB173) & 39.3 & 0.27 & 15.5 & 4.9 & 0.08 & 0.02 & -0.09 & 0.00 \\
\hline 42301 (2001 UR163) & 51.9 & 0.28 & 0.8 & 4.2 & -0.01 & 0.13 & 0.01 & 0.05 \\
\hline 42355 Typhon (2002 CR46) & 37.7 & 0.54 & 2.4 & 7.5 & 0.31 & 0.17 & 0.05 & 0.10 \\
\hline 44594 (1999 OX3) & 32.5 & 0.46 & 2.6 & 7.4 & 0.31 & 0.15 & 0.21 & 0.16 \\
\hline 47171 (1999 ТС36) & 39.7 & 0.23 & 8.4 & 4.9 & 0.08 & 0.04 & -0.08 & 0.00 \\
\hline 47932 (2000 GN171) & 39.2 & 0.28 & 10.8 & 6.0 & -0.05 & 0.08 & 0.04 & 0.03 \\
\hline 50000 Quaoar (2002 LM60) & 43.3 & 0.04 & 8.0 & 2.6 & 0.29 & 0.01 & 0.07 & 0.00 \\
\hline 52872 Okyrhoe (1998 SG35) & 8.3 & 0.31 & 15.7 & 10.9 & 0.08 & 0.05 & -0.02 & 0.03 \\
\hline 54598 Bienor (2000 QC243) & 16.6 & 0.20 & 20.7 & 7.5 & 0.07 & 0.07 & -0.00 & 0.03 \\
\hline 55565 (2002 AW197) & 47.1 & 0.13 & 24.4 & 3.4 & 0.04 & 0.03 & 0.03 & 0.00 \\
\hline 55636 (2002 TX300) & 43.5 & 0.12 & 25.8 & 3.2 & 0.91 & 0.01 & -1.47 & 0.24 \\
\hline 55637 (2002 UX25) & 42.9 & 0.14 & 19.4 & 3.7 & 0.03 & 0.04 & -0.02 & 0.00 \\
\hline 55638 (2002 VE95) & 39.6 & 0.29 & 16.3 & 5.6 & 0.14 & 0.02 & 0.05 & 0.00 \\
\hline 65489 Ceto (2003 FX128) & 99.8 & 0.82 & 22.3 & 6.3 & 0.17 & 0.06 & -0.10 & 0.03 \\
\hline 83982 Crantor (2002 GO9) & 19.4 & 0.28 & 12.8 & 8.8 & 0.16 & 0.05 & -0.07 & 0.03 \\
\hline 84522 (2002 TC302) & 55.7 & 0.30 & 35.0 & 3.8 & 0.13 & 0.08 & 0.07 & 0.03 \\
\hline 84922 (2003 VS2) & 39.6 & 0.08 & 14.8 & 4.1 & 0.09 & 0.02 & -0.03 & 0.00 \\
\hline 90482 Orcus (2004 DW) & 39.2 & 0.23 & 20.6 & 2.3 & 0.44 & 0.01 & -0.36 & 0.03 \\
\hline 90568 (2004 GV9) & 41.8 & 0.07 & 22.0 & 4.0 & 0.05 & 0.05 & -0.04 & 0.03 \\
\hline 95626 (2002 GZ32) & 23.0 & 0.22 & 15.0 & 7.0 & 0.01 & 0.03 & 0.05 & 0.00 \\
\hline 119951 (2002 KX14) & 38.7 & 0.05 & 0.4 & 4.4 & -0.14 & 0.22 & -0.08 & 0.06 \\
\hline 120061 (2003 CO1) & 20.7 & 0.47 & 19.8 & 8.9 & -0.01 & 0.02 & 0.08 & 0.00 \\
\hline 120132 (2003 FY128) & 49.2 & 0.25 & 11.8 & 4.9 & 0.13 & 0.28 & -0.11 & 0.17 \\
\hline 120178 (2003 OP32) & 43.3 & 0.10 & 27.1 & 3.6 & 1.01 & 0.00 & 10.18 & 0.24 \\
\hline 120348 (2004 TY364) & 39.1 & 0.06 & 24.8 & 4.5 & 0.06 & 0.02 & 0.04 & 0.00 \\
\hline 127546 (2002 XU93) & 66.6 & 0.69 & 77.9 & 8.0 & 0.07 & 0.23 & 0.09 & 0.10 \\
\hline 134860 (2000 OJ67) & 43.0 & 0.02 & 1.1 & 6.1 & 0.80 & 0.33 & -0.84 & 0.24 \\
\hline 136108 Haumea (2003 EL61) & 43.0 & 0.20 & 28.2 & 0.2 & 0.66 & 0.00 & -0.40 & 0.00 \\
\hline 136472 Makemake (2005 FY9) & 45.4 & 0.16 & 29.0 & -0.4 & -1.68 & 0.05 & -0.22 & 0.00 \\
\hline 145451 (2005 RM43) & 92.2 & 0.62 & 28.7 & 4.4 & 0.32 & 0.05 & -0.20 & 0.03 \\
\hline 145452 (2005 RN43) & 41.7 & 0.03 & 19.2 & 3.9 & -0.06 & 0.03 & -0.01 & 0.00 \\
\hline 145453 (2005 RR43) & 43.5 & 0.14 & 28.5 & 4.0 & 1.01 & 0.00 & 5.61 & 0.24 \\
\hline 145486 (2005 UJ438) & 17.7 & 0.53 & 3.8 & 10.7 & 0.02 & 0.09 & 0.03 & 0.03 \\
\hline 174567 (2003 MW12) & 45.7 & 0.14 & 21.5 & 3.4 & -0.00 & 0.05 & -0.05 & 0.01 \\
\hline 202421 (2005 UQ513) & 43.5 & 0.14 & 25.7 & 3.4 & 0.09 & 0.04 & 0.03 & 0.03 \\
\hline 208996 (2003 AZ84) & 39.5 & 0.18 & 13.5 & 3.6 & 0.23 & 0.06 & -0.10 & 0.03 \\
\hline 225088 (2007 OR10) & 67.1 & 0.50 & 30.7 & 1.7 & 0.34 & 0.07 & 0.06 & 0.05 \\
\hline 229762 (2007 UK126) & 74.2 & 0.49 & 23.4 & 3.4 & 0.01 & 0.03 & -0.11 & 0.03 \\
\hline 248835 (2006 SX368) & 22.3 & 0.46 & 36.3 & 9.5 & 0.10 & 0.03 & 0.13 & 0.03 \\
\hline (2000 PE30) & 54.5 & 0.34 & 18.4 & 6.1 & 0.56 & 0.52 & 0.51 & 0.24 \\
\hline (2004 NT33) & 43.6 & 0.15 & 31.2 & 4.4 & 0.07 & 0.07 & 0.00 & 0.03 \\
\hline (2004 PG115) & 91.0 & 0.60 & 16.3 & 4.9 & 0.07 & 0.03 & 0.07 & 0.00 \\
\hline (2005 QU182) & 114.0 & 0.67 & 14.0 & 3.4 & -0.17 & 0.05 & 0.06 & 0.00 \\
\hline (2007 UM126) & 12.9 & 0.34 & 41.7 & 10.1 & -0.01 & 0.06 & 0.20 & 0.03 \\
\hline
\end{tabular}


temperatures of $50 \mathrm{~K}$ and created a model spectrum assuming grain sizes of $50 \mu \mathrm{m}$ using the method of Hapke (1993). Our goal is to have a single representative water ice spectrum to use in our parameterized fit rather than to fit for additional parameters of the water ice spectrum such as grain size or temperature. The sloped continuum is constrained to have a reflectance of 0.49 at $1.74 \mu \mathrm{m}$ to match that of the water ice component that we used. Without a direct measurement of the true albedo of the object, however, the actual albedo of the continuum component is unconstrained, but the parameter $f_{\text {water }}$ approximates how much of the spectrum is being modeled by water ice versus how much by continuum. We will use the term "fraction of water ice the in the spectrum" to describe this parameter, but we note again that there is no unique way to convert $f_{\text {water }}$ into a fraction of water ice on the surface.

We estimate the uncertainties in the spectra by median smoothing each spectrum with an 11 pixel wide box and then calculating the standard deviation of the difference between the original spectrum and the smoothed spectrum. We then perform a $\chi^{2}$ minimization to find the best-fit values for the two parameters, $f_{\text {water }}$, the fraction of water ice in the spectrum, and $m_{\text {cont }}$ the slope of the continuum using only data between 1.45 and $1.8 \mu \mathrm{m}$ and 1.95 and $2.3 \mu \mathrm{m}$, where atmospheric transmission and spectroscopic throughput are highest. After minimizing, we create a grid of $f_{\text {water }}$ and $m_{\text {cont }}$ and find $\chi^{2}$ for each point. Formally, the $1 \sigma$ errors on the parametric fitting are where $\chi^{2}$ increases from its minimum to 1 above the minimum. We find, however, that, by eye, these uncertainties appear unreasonably small, which is not unexpected. Error bars derived from a $\chi^{2}$ minimization will only give correct results if the model perfectly describes the data and the error bars on the data are perfectly Gaussian. Neither of these conditions is likely to be true. After extensive experimentation with fitting by hand and examining the $\chi^{2}$ values in spectra where we believe we do and do not credibly detect water ice, we have adopted errors on our parameters to include the regions where $\chi^{2}$ increases to 5 above the minimum. While it is difficult to assign a rigorous statistical meaning to the uncertainties, we believe them to be the best representation of credible uncertainties. Full fit parameters of all objects in our sample are given in Table 2, and the fits to the new spectra are shown in Figure 1.

\section{THE MID-SIZED KUIPER BELT OBJECTS}

Of the 57 objects in the spectral survey, most have uncertainties in $f_{\text {water }}$ clustered below 0.1 , but 10 have significantly larger errors. We deem those spectra too noisy for reliable analysis and discard those 10 objects from further consideration. In Figure 2, we show the fraction of water ice in the spectrum as a function of absolute magnitude for the remaining 47 objects of the sample. A trend of higher water ice absorption on the intrinsically brightest objects is clearly present. Below an absolute magnitude of $H=3$, all KBOs have significant water ice absorption, which generally increases with lower absolute magnitude. Above an absolute magnitude of $H=4.5$, no trend with size is apparent. A similar result is shown in Barucci et al. (2011). While such a correlation of absolute magnitude and presence of water ice might be expected simply from the increased albedo of objects with more water ice on their surfaces, Spitzer radiometry has shown that, discounting the Haumea family members, the objects with the largest water ice absorption are indeed the largest objects, and not just the objects with highest albedos (Stansberry et al. 2008).

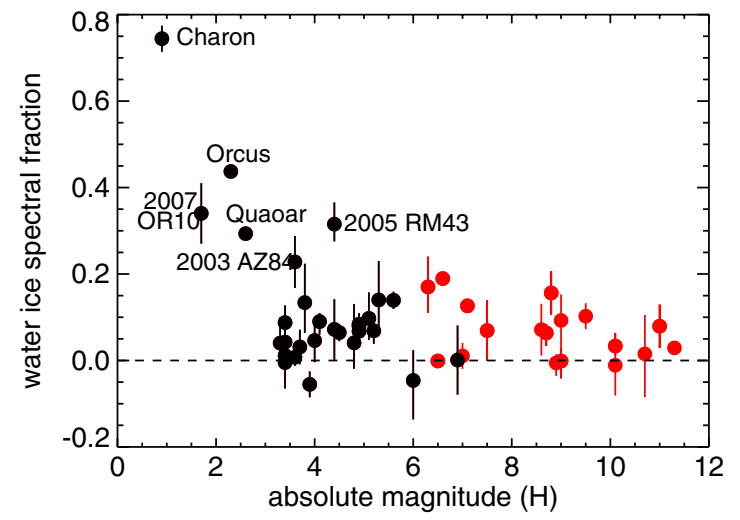

Figure 2. Water ice absorption as a function of absolute magnitude. Objects with semimajor axis outside of $30 \mathrm{AU}$ are shown in black, while those inside of $30 \mathrm{AU}$ are shown in red. Absolute magnitudes are taken from the Minor Planet Center and have typical random errors below 0.3 mag.

(A color version of this figure is available in the online journal.)

The trend of increasing surface water ice fraction with increasing size for the largest objects is in marked contrast to the trend of increasing density - and thus decreasing bulk water ice fraction-with increasing size (Brown 2012).

Desch et al. (2009) and Delsanti et al. (2010) considered the possibility of surface water flow on Charon and on Orcus-two of the objects with the largest fraction of water ice on their surfaces - and concluded that liquid water interiors could have been present in the past and resulted in water or water-ammonia flows through cracks to the surface. The detection of ammonia on Charon (Brown \& Calvin 2000; Dumas et al. 2001; Cook et al. 2007; Merlin et al. 2010) and likely also on Orcus (de Bergh et al. 2005; Barucci et al. 2008; Carry et al. 2011) supports this idea. While modeling has yet to explore much parameter space, it seems clear that this type of cryovolcanic activity and the amount of water emplaced on the surface would increase with increasing size of the object.

It is instructive to note that simple order-of-magnitude calculations support the idea that somewhere around a diameter of $500 \mathrm{~km}$ liquid water could have been an important component of the early object. For example, if all of the accretional gravitational potential energy were converted into heat, an object with a 1:2 ice-rock mass mix with a diameter of $700 \mathrm{~km}$ would have enough energy to melt all of its ice. Similarly, assuming initial chondritic heating rates (including no short-lived radionuclides) for the rock fraction (Castillo-Rogez et al. 2007), an object $500 \mathrm{~km}$ in diameter would have enough radioactive heating to melt all of its ice in only $60 \mathrm{kyr}$. Neither of these scenarios represents a realistic assumption about heat flow in these bodies, but they provide interesting limits which point to the $500 \mathrm{~km}$ size as a potentially important transition size.

Simply having cryovolcanic flow on the surface in the past does not guarantee that the ice would still be visible today. In the scenarios of Desch et al. (2009) and Delsanti et al. (2010), the cryovolcanic flow occurs as the liquid interior begins to freeze and squeeze water out through cracks, a process which would necessarily be delayed. Desch et al. (2009) proposes that the cryovolcanic flows on Charon are actually recent, but Schubert et al. (2010) suggest that the physical parameters they require for current cryovolcanism are implausible. From the simple existence of water ice on the surface, however, there is no need to invoke current or even recent cryovolcanism; the very fresh water ice on the surface of Haumea and its collisional 
family (Brown et al. 2007) combined with dynamical modeling which shows that the surfaces have likely been exposed for most of the age of the solar system (Ragozzine \& Brown 2007; Levison et al. 2008) demonstrate that fresh water ice surfaces can be preserved on billion year timescales in the outer solar system.

If the water ice on the surfaces of the medium-sized KBOs is indeed from cryovolcanic flows, and if the early interior oceans of these medium-sized KBOs were of similar composition, we would expect that, like Charon and apparently Orcus, all of the objects would also have ammonia extruded onto their surfaces with the water ice. Some of the larger and colder objects retain a tiny amount of methane, whose strongest absorption feature is in the same location as that of ammonia, so confirmation of ammonia absorption features is difficult. The objects smaller than Orcus currently do not have spectra with sufficient signalto-noise to be able to test for the presence of ammonia.

\section{SMALLER OBJECTS}

Many objects with absolute magnitude $H>3$ (corresponding approximately to a diameters of $800 \mathrm{~km}$ and smaller; Stansberry et al. 2008) are consistent with having little or no water ice detected on their surface, but almost all of these objects have $f_{\text {water }}>0$. Photon noise or systematic error from telluric correction - the two largest sources of uncertainty in the data-could not produce a bias toward positive values of $f_{\text {water }}$. Indeed, we regard the nearly complete lack of objects which are constrained to negative values of $f_{\text {water }}$ within their uncertainties as an indication of the robustness of our method. We conclude, therefore, that even the low level of water ice fraction detected in the majority of the objects is a real indication that water ice is common within the spectra of the smallest measurable objects.

To date, no trends have been found or explanations proposed for the low levels of water ice on the smaller objects. Barkume et al. (2006) found no simple correlation with color or orbital parameters; a recent analysis of a large sample of spectra (Barucci et al. 2011) similarly found no correlations.

Recently, however, Fraser \& Brown (2012, hereafter the H/WTSOSS survey) have used a large visible-infrared photometric survey from the Hubble Space Telescope (HST) to suggest that - when the cold-classical KBOs and Haumea family members are excluded from the sample-small KBOs and centaurs consist of two distinct compositional families. Each family is described by a mixture between a common dark neutrally colored substance and either a slightly red (for the neutral family) or very red (for the red family) component. Both red components are characterized by a decrease in reflectivity between 1.38 and $1.53 \mu \mathrm{m}$, which is consistent with a contribution from water ice in addition to whatever is causing the red coloration. In order to analyze if such a trend is visible in our measured water ice fraction, we examine the water ice spectral fraction of all objects with $f_{\text {water }}<0.2$ (all KBOs with $f_{\text {water }}>0.2$ appear to have surface characteristics influenced by their larger size and thus we discard them from the sample), as a function of color (Figure 3). We use optical photometry from the MBOSS compilation $^{4}$ to construct a spectral gradient using the method of Hainaut \& Delsanti (2002), but only using the $B, V, R$, and $I$ colors, to more closely match the H/WTSOSS measurements. The spectral calculated spectra gradient for the full sample can be found in Table 2. Colors have been measured for 29 of our $41 f_{\text {water }}<0.2$ objects. Similar to previous analysis, we find no

\footnotetext{
4 Available at http://www.eso.org/ohainaut/MBOSS/.
}

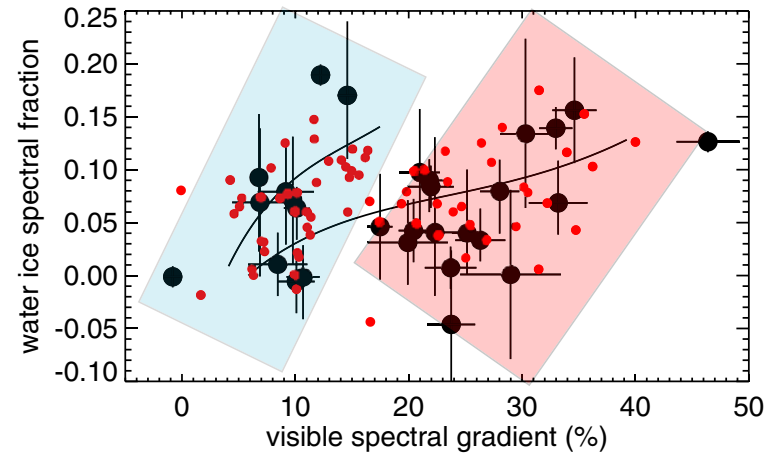

Figure 3. Water ice absorption fraction as a function of spectral color gradient. Black points with error bars show the spectral data and colors derived from visible photometry. The red points without error bars (typical uncertainties are smaller than those of the spectral data) are from the H/WTSOSS photometric survey of Fraser \& Brown (2012) and have been transformed to these variables. The two lines show the mixing models which best fit the three-color H/WTSOSS data. The blue and red shading show the neutral and the red families of objects. (A color version of this figure is available in the online journal.)

simple trend between water ice absorption and color. However, if we examine the neutral objects (which we empirically define as spectral gradient less than $17 \% / 100 \mathrm{~nm}$ ) and red objects (spectral gradient greater than $17 \% / 100 \mathrm{~nm}$ ) separately we begin to see the trend which would be predicted by the H/WTSOSS survey: within each color group, water ice absorption becomes stronger as the objects become redder. The uncertainties in the water ice absorption are sufficiently high that obtaining robust statistics is impossible, though for the red objects, a rank correlation test shows that there is a positive correlation between the color and the fraction of water ice absorption at the $87 \%$ confidence level. Measurement of the colors of the 12 objects for which no such measurements yet exist will help to better define the behavior of the data.

To further examine the relationship between these spectral results and the photometric results of the H/WTSOSS survey we derive an approximate transformation from the HST photometric colors to our color gradient-water ice absorption system. First, we use the 41 objects which have both ground-based spectral gradient measurements and H/WTSOSS F606W and F814W photometry to derive an empirical second-order polynomial transformation from [F606W]-[F814W] color to spectral gradient. We then model a series of water ice plus continuum spectra with increasing values of $f_{\text {water }}$ and we measure the equivalent [F139M]-[F152M] H/WTSOSS color for each of these spectra. The relationship between $\mathrm{H} / \mathrm{WTSOSS}$ color and $f_{\text {water }}$ is nearly linear, but we fit a second-order polynomial to more precisely define our transformation.

Using these transformations, we show the Fraser \& Brown (2012) mixing models which provide the best fit to the H/ WTSOSS three-color data. The results show that not only do the mixing models qualitatively suggest that the red ends of the two color families should have more water ice absorption, but, remarkably, the mixing models also predict the overall magnitude of the water ice absorption correctly. To further examine the correlation between the H/WTSOSS and the spectroscopic data, we show all of the H/WTSOSS data for $H>$ 5.5 (this was the absolute magnitude that Fraser \& Brown (2012) chose to analyze because of the large number of both KBOs and centaurs fainter than this value) non-cold-classical and nonHaumea family KBOs converted to our visible gradient-water absorption system. The photometric measurements of water 
ice follow the same general behavior and occupy the same range as the spectroscopic measurements. (Note that both the photometric and spectroscopic results suggest that the common end member of the mixing models is likely redder than suggested by Fraser \& Brown (2012). A possible reason for this discrepancy is that the end members for the mixing model were restricted in color space to be within the range of measured object colors, which appears unlikely to be true.) This remarkable correspondence between the spectroscopic and photometric data suggests that both data sets are viewing the same phenomenon; the H/WTSOSS data truly do provide a measurement of water ice absorption, and the spectroscopic data support the same dual mixing model interpretation as the H/WTSOSS data.

More remarkably, this interpretation suggests that we can now identify another of the components of the mixing model. Fraser \& Brown (2012) suggested that the common end member is consistent with the colors of hydrated silicates. These data suggest that the two other end members both contain water ice as part of their constituents. Water ice cannot be the only constituent, however, as both of these end members are more red than water ice. It is clear, then, that the water ice must be mixed with some other component in the end members to provide the red color. Interestingly, the water ice fraction in the end member must remain nearly constant even as the end member is mixed with varying amounts of the silicate-like end member.

\section{METHANOL}

Irradiated hydrocarbons appear to be a natural explanation for the red materials in the two red end members. In the evaporation gradient hypothesis of Brown et al. (2011b), surfaces in the early Kuiper Belt bifurcate into those cold enough to retain methanol and those too hot for methanol to remain on the surface. In this hypothesis one end member would be an irradiated mixture of $\mathrm{CO}_{2}$ and water ice, while the other would be an irradiated mixture of water ice, $\mathrm{CO}_{2}$, with methanol added, causing a redder color (Brunetto et al. 2006).

Methanol is the only other ice that has been suggested to be present in the spectra of small KBOs or centaurs. An absorption feature around $2.27 \mu \mathrm{m}$ was first observed on the centaur Pholus (Davies et al. 1993) and later suggested to be due to methanol or a similar light hydrocarbon (Cruikshank et al. 1998). Subsequent observations suggested that the KBOs 1998 GQ21 and 2002 VE95 had spectra very similar to that of Pholus, including the $2.27 \mu \mathrm{m}$ absorption (Barkume et al. 2006; Barucci et al. 2011). The objects Pholus, 1996 GQ21, and 2002 VE95 are three of the four reddest objects in our sample. Recently, it has been suggested that many more objects might have these $2.27 \mu \mathrm{m}$ absorption features though they do not necessarily share the other characteristics of the surface of Pholus (moderate water ice absorption, very red color; Barucci et al. 2011).

Robust detection of narrow features at this wavelength is difficult for these faint objects. Nonetheless, we believe that a statistical assessment might still discern trends in methanol absorption even if the individual spectra have less reliable results. We therefore model all of the spectra allowing an additional methanol ice component, where the methanol spectrum is taken from Cruikshank et al. (1998). We define the methanol ice fraction, $f_{\text {methanol }}$ similarly to $f_{\text {water }}$ for water ice and derive the uncertainty in the same manner. Results for all objects in the extended sample are listed in Table 2. To better analyze the data we first discard all of the measurements for which the uncertainties in $f_{\text {methanol }}$ are greater than the median uncertainty

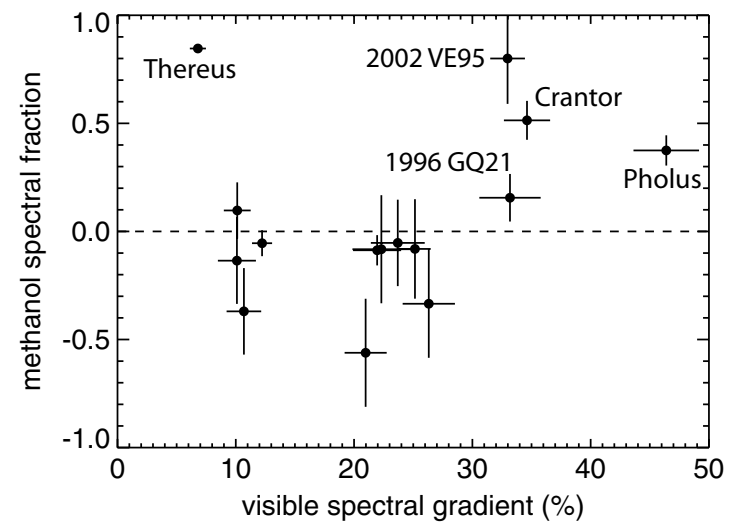

Figure 4. Methanol ice absorption fraction as a function of spectral color gradient. The red family shows a positive correlation between color and methanol absorption and color at the $99.8 \%$ confidence level, as would be predicted by the methanol evaporation model for KBO coloration. In the blue family, methanol is only detected on the centaur Thereus, but higher quality spectra suggest that this detection is spurious.

in our restricted sample. We then examine the methanol absorption fraction as a function of object color (Figure 4). The red family of objects (with spectral gradient greater than $17 \%$ / $100 \mathrm{~nm}$ ) shows a positive correlation of methanol absorption with color at the $98.8 \%$ confidence level. The blue family of objects shows no such correlation. Indeed, the only blue object on which methanol is potentially detected at a significant level-the centaur Thereus - was examined with much higher signal-to-noise by Merlin et al. (2005), who strongly rule out any methanol absorption at the level suggested here. While it is possible that the spectrum is strongly rotationally variable, we suspect, instead, that our measurement of methanol is simply spurious. Further observations of this object are warranted.

Of the objects in the red population, all four for which our method reports robust detections of methanol have previously been identified as containing methanol-like absorption features-Pholus (Cruikshank et al. 1998), 1996 GQ21 (Barucci et al. 2011), 2002 VE95 (Barkume et al. 2006), and Crantor (Alvarez-Candal et al. 2007).

The appearance of methanol conforms to the expectation of the evaporation gradient model (Brown et al. 2011b) combined with the H/WTSOSS mixing model. No clear detections of methanol are made in the blue population, while in the red population methanol is only clearly detected on the reddest end, where the mixing model predicts the most ices.

\section{DISCUSSION}

The spectroscopic and photometric analysis here shows that water ice in the Kuiper Belt comes through three separate distinct processes. Nearly pure water ice is seen only on Haumea and on Haumea's collisional family (and satellites) and must be related to the impact that created the family. The small sizes of the family members, coupled with the low densities of Haumea's satellites (Ragozzine \& Brown 2009), suggest that these bodies are undifferentiated and nearly pure water ice throughout. Such pure water ice surfaces could be formed if the collisional family is fragments of a nearly pure icy mantle in a early differentiated proto-Haumea. Fragments from the crust of the proto-Haumea, which would presumably show a darker hydrocarbon irradiated surface, have not been identified, but might be present. Crust fragments will be rare compared to mantle fragments, however. 
KBOs with absolute magnitudes smaller than $H=3$ (diameters $\gtrsim 800 \mathrm{~km}$ ) have increasingly abundant water ice on their surfaces, presumably as a result of cryovolcanism at some point in the (possibly quite distant) past. Some objects between $H=3$ and $H=4.5$ also have abundant water ice, while none with $H>4.5$ have as much. No obvious characteristics separate the more water ice-rich objects 2003 AZ84 and 2005 RM43 from their similarly sized less-icy objects; differences in formation location, density, or evolutionary history are obvious candidates for these differences, but verification will be difficult.

Smaller KBOs and centaurs show a systematic trend of water ice and color that corresponds to the trends found in the $\mathrm{H} /$ WTSOSS photometric survey. When Haumea family members and cold-classical KBOs are excluded from the population, the remaining objects form two color families, each of which can be described by a mixing model. From the H/WTSOSS photometric data on small objects, Fraser \& Brown (2012) suggested that the mixing component that causes an apparent absorption at $1.54 \mu \mathrm{m}$ could be water ice. The spectroscopic data on larger objects support this suggestion.

We now have possible identifications for two of the four major components of the mixing model. Fraser \& Brown (2012) suggested that the component that is common to both families is consistent with many common hydrated silicates. For the neutral family, this component is mixed with a second component which is a mixture of a slightly red material and water ice (thus the "neutral" family is, in fact, slightly red). For the red family, the common component is mixed with a second component which is a mixture of a very red material and water ice.

The difference between the slightly red and very red materials is possibly caused by the absence or presence of methanol ice on the surface of the object during early irradiation (Brown et al. 2011b). Objects that formed inside of $\sim 20 \mathrm{AU}$ in a compact early solar system would have been too hot to retain surface methanol for an amount of time long enough for methanol irradiation to affect the coloration. Outside of $20 \mathrm{AU}$, objects are cold enough that methanol remains present, possibly explaining the redder component of the red family. This suggestion is supported by the observation that methanol is only robustly detected on the very reddest members of the red population.

This research has been supported by the grant NNX09AB49G from the NASA Planetary Astronomy program. Some of the data presented here were obtained at the W. M. Keck Observatory, which is operated as a scientific partnership among the California Institute of Technology, the University of California, and the National Aeronautics and Space Administration. The Observatory was made possible by the generous financial support of the W. M. Keck Foundation. Support for program HST-GO-11644.01-A was provided by NASA through a grant from the Space Telescope Science Institute, which is operated by the Association of the Universities for Research in Astronomy, Inc., under NASA contract NAS 5-26555.

\section{REFERENCES}

Alvarez-Candal, A., Barucci, M. A., Merlin, F., Guilbert, A., \& de Bergh, C. 2007, A\&A, 475, 369

Barkume, K. M., Brown, M. E., \& Schaller, E. L. 2006, ApJ, 640, L87

Barkume, K. M., Brown, M. E., \& Schaller, E. L. 2008, AJ, 135, 55

Barucci, M. A., Alvarez-Candal, A., Merlin, F., et al. 2011, Icarus, 214, 297

Barucci, M. A., Merlin, F., Guilbert, A., et al. 2008, A\&A, 479, L13

Brown, M. E. 2000, AJ, 119, 977

Brown, M. E. 2012, Annu. Rev. Earth Planet. Sci., 40, 467

Brown, M. E., Barkume, K. M., Ragozzine, D., \& Schaller, E. L. 2007, Nature, 446, 294

Brown, M. E., Burgasser, A. J., \& Fraser, W. C. 2011a, ApJ, 738, L26

Brown, M. E., \& Calvin, W. M. 2000, Science, 287, 107

Brown, M. E., Schaller, E. L., \& Fraser, W. C. 2011b, ApJ, 739, L60

Brown, R. H., Cruikshank, D. P., \& Pendleton, Y. 1999, ApJ, 519, L101

Brown, R. H., Cruikshank, D. P., Pendleton, Y., \& Veeder, G. J. 1998, Science, 280, 1430

Brunetto, R., Barucci, M. A., Dotto, E., \& Strazzulla, G. 2006, ApJ, 644, 646

Carry, B., Hestroffer, D., DeMeo, F. E., et al. 2011, A\&A, 534, A115

Castillo-Rogez, J. C., Matson, D. L., Sotin, C., et al. 2007, Icarus, 190, 179

Cook, J. C., Desch, S. J., Roush, T. L., Trujillo, C. A., \& Geballe, T. R. 2007, ApJ, 663,1406

Cruikshank, D. P., Roush, T. L., Bartholomew, M. J., et al. 1998, Icarus, 135 , 389

Davies, J. K., Sykes, M. V., \& Cruikshank, D. P. 1993, Icarus, 102, 166

de Bergh, C., Delsanti, A., Tozzi, G. P., et al. 2005, A\&A, 437, 1115

Delsanti, A., Merlin, F., Guilbert-Lepoutre, A., et al. 2010, A\&A, 520, A40

Desch, S. J., Cook, J. C., Doggett, T. C., \& Porter, S. B. 2009, Icarus, 202, 694

Dumas, C., Terrile, R. J., Brown, R. H., Schneider, G., \& Smith, B. A. 2001, AJ, 121,1163

Fraser, W. C., \& Brown, M. E. 2012, ApJ, 749, 33

Grundy, W. M., \& Schmitt, B. 1998, J. Geophys. Res., 103, 25809

Guilbert, A., Alvarez-Candal, A., Merlin, F., et al. 2009, Icarus, 201, 272

Hainaut, O. R., \& Delsanti, A. C. 2002, A\&A, 389, 641

Hapke, B. 1993, Theory of reflectance and emittance spectroscopy (Topics in Remote Sensing; Cambridge: Cambridge Univ. Press)

Jewitt, D. C., \& Luu, J. X. 2001, AJ, 122, 2099

Levison, H. F., Morbidelli, A., Vokrouhlický, D., \& Bottke, W. F. 2008, AJ, 136, 1079

Matthews, K., \& Soifer, B. T. 1994, Exp. Astron., 3, 77

Merlin, F., Barucci, M. A., de Bergh, C., et al. 2010, Icarus, 210, 930

Merlin, F., Barucci, M. A., Dotto, E., de Bergh, C., \& Lo Curto, G. 2005, A\&A, 444, 977

Ragozzine, D., \& Brown, M. E. 2007, AJ, 134, 2160

Ragozzine, D., \& Brown, M. E. 2009, AJ, 137, 4766

Romanishin, W., \& Tegler, S. C. 2005, Icarus, 179, 523

Schaller, E. L., \& Brown, M. E. 2007, ApJ, 659, L61

Schubert, G., Hussmann, H., Lainey, V., et al. 2010, Space Sci. Rev., 153, 447

Stansberry, J., Grundy, W., Brown, M., et al. 2008, in The Solar System Beyond Neptune, ed. M. A. Barucci, H. Boehnhardt, D. P. Cruikshank, \& A. Morbidelli (Tucson, AZ: Univ. Arizona Press), 161 